\title{
Then and Now
}

\section{Gilbert I. Martin, MD}

I have pointed out in many of these columns over the years that to understand the present and plan for the future, we must appreciate the past. There is a wonderful book written by William Silverman that should be required reading for all personnel caring for mothers and newborns. This treatise, entitled Where's the Evidence, was published by Oxford University Press in 1998 and is a series of essays, written in the past, with commentaries on what has transpired over the ensuing years. I have taken the liberty of using this approach to present a topic offered to our readers in 1984 with subsequent developments over the past 15 years.

\section{4: Resuscitation at Birth: Who to Train ${ }^{1}$}

Traditionally, pediatric residents are trained to resuscitate newborn infants in the delivery room. Over a period of time, most become proficient at this task. Indeed, the ability to intubate is required for true admission into the fraternity (sorority) of "physicianhood." And yet, the ability to expertly resuscitate, including intubation when necessary, does not require an MD degree, or any other degree for that matter. It does require theoretical and practical training and regular application of the skills acquired.

Once the average pediatric resident graduates and becomes an attending, opportunities to practice and maintain these acquired skills decrease precipitously. Many pediatric residents report that the need to intubate an infant at birth occurs only once or twice a year after their graduation from pediatric training, a year in which a disproportionate percentage of their patients are newborns. The years that follow generally provide even less exposure, thereby permitting further deterioration of their resuscitation skills. From time to time, "refresher" courses are provided with mannequin or cat models. Nonetheless, the true expert is the person who practices his or her art regularly. Cardiac surgeons who perform only two or three coronary artery by-pass grafts per year, or general surgeons who perform only two or three cholecystectomies per year, or cardiologists who perform only two or three cardiac catherizations per year will not be granted hospital privileges or will have their privileges revoked. Why should the difficult task of expert neonatal resuscitation be viewed any differently?

If pediatric residents are the wrong group to train, which is the right group? Many if not most infants who require resuscitation in the delivery room require it unexpectantly. Therefore, every delivery service should have a designated resuscitator in the deliv- ery room area, 24 hours a day. A delivery room nurse would fill this role most easily, although other disciplines such as respiratory care may be adequate. Sufficient numbers of nurses need to be trained to provide one nurse resuscitator on each and every shift. Trained personnel should be limited in number so as not to dilute the available experience. Even relatively small delivery services will have enough infants requiring resuscitation to allow each trained nurse several opportunities per month to practice and maintain his or her skills. Physicians would then be deprived of the glory, but birth asphyxia would get to take a holiday more often.

\section{Resuscitation at Birth: Who to Train}

The ideas noted in this 1984 editorial were certainly predictive of what has developed today. There are many reasons for these changes, and in certain areas, we are not yet complete. The entire pediatric residency experience in the neonatal intensive care unit (NICU) has been shortened considerably. Under current standards, 3 months are spent in the NICU; therefore, the resident has fewer opportunities to perform intubations. In the past, senior residents were the first to the delivery room for a resuscitation. Now other members of the "team" are more proficient. Upon graduation to practice, their experience has been dampened considerably. Once in practice, several scenarios take place. With many managed care practices, pediatricians are encouraged to see patients in their offices and to spend as little time as possible taking care of patients in the hospital. Thus, "hospitalists" or "neonatologists" have taken up much of this responsibility. Most obstetricians would prefer that there be a designated neonatologist available to the delivery room at all times. In hospitals in which the neonatologist "sleeps in," this is possible. However, most hospitals delivering babies do not have this capability; therefore, the neonatologist will not be an option.

Other personnel must be trained. In the ideal situation, nurses are the obvious choice. However, except for tertiary hospitals, the personnel required and the number of "intubating experiences" would not be adequate. Second, to assign a nurse already taking care of two or three other infants to this role would be difficult. Third, in tertiary centers, which nurse would go on transports, if necessary? A neonatal nurse practitioner would be perfect in this role, but they are usually part of the more specialized NICU. Therefore, in many situations, the respiratory therapist has assumed the role of "intubator." Even primary hospitals today have respiratory therapists in house, and all hospitals with a delivery service need to have this as a "policy."

Because, in medicine, we strive for the "ideal," what would this 
concept mean? There should be a "neonatal resuscitation team" available for all deliveries. This team should be in house, and there needs to be a back up group if the team is called for a transport. This team should consist of at least a nurse and respiratory therapist skilled in neonatal resuscitation with a neonatologist available as back up.

Our task has been made easier by the American Academy of Pediatrics and the American Heart Association with the production of the Neonatal Resuscitation Program. The fourth edition of the Neonatal Resuscitation Program manual is forthcoming. This program has become the most widely used course of the American Academy of Pediatrics around the world. Proficiency in neonatal resuscitation must be required for physicians, nurses, and respiratory therapists working in hospitals that deliver babies.

We must realize that the number of times that resuscitation is necessary will not decrease. As larger numbers of very low birth weight infants are born, the number of resuscitations will increase. If we agree that the opportunities are there, and that the dedication and expertise is present; what is the dilemma?

\section{Cost, Cost, Cost!!!!!}

It takes money to pay for the training and the hiring of the personnel that are necessary to perform these responsibilities. Hospitals are hav- ing a difficult time in this era of managed care and are not able to secure funding. Reimbursement for attending deliveries and performing resuscitation is poor at best, and the neonatologist cannot be in all places at once, and might prefer that another group assume this role. The obstetrician and anesthesiologist in the delivery room or surgical suite would much prefer that a pediatrician/neonatologist be present for the delivery, for they know that as physicians, they have the ultimate responsibility. The payers (both private and government) must adjust their goals accordingly to allow for improvement in this most critical aspect of newborn care. No one would argue that the first few minutes of life can determine the productively of the individual for the future.

The editorial written in 1984 was predictive of our current state in many ways but did not consider the fiscal consequences. I look forward to writing another editorial in 2015 on the same subject to determine whether or not we have become progressive and proactive or have simply eliminated the problem.

\section{References}

1. Pomerance JJ, Martin GI. Resuscitation at birth: who to train. J Calif Perinat Assoc $1984 ; 2: 13$ 